\title{
Generalised Absorption Efficiency of Yagi-Uda Antennas
}

\author{
Morteza Shahpari, David V. Thiel \\ Center for Wireless Monitoring and Applications, Griffith University, Brisbane, Australia \\ morteza.shahpari@ieee.org, d.thiel@griffith.edu.au
}

\begin{abstract}
The generalised absorption efficiency is the ratio of the all-spectrum absorbed to scattered energies. Although often estimated as 0.5 , it should be rigorously calculated for the accurate estimation of fundamental limits. Many antennas, as tuned in the resonant frequency, absorb as much power as they scatter. One of the interesting properties of the YagiUda antennas is more absorption rather scattering. Absorption, scattering, and extinction cross sections of a typical Yagi-Uda antenna are reported in this paper to study the generalised absorption efficiency for this antenna type.
\end{abstract}

\section{INTRODUCTION}

Generalised absorption efficiency $\check{\eta}_{a}$ was introduced for the first time in [1] for the calculation of a fundamental limit of $D / Q$ ratio. Generally, $\check{\eta}_{a}$ is estimated as 0.5 for minimum scattering antennas (MSA) as well as antennas with a dominant resonance in their absorption spectrum [2]. However, a proper calculation of the upper bound on $D / Q$ ratio needs a priori knowledge of the $\check{\eta}_{a}$ [3].

Discussions are characterized since 2002 by arguments on the equivalent circuit of receiving antennas [4] as well as total absorbed and scattered power [5]. Yagi-Uda antennas have been studied extensively in the discussions due to their special ability to absorb more power from the incoming electromagnetic wave rather than scattering (absorption efficiency $\left.\eta_{a}>0.5\right)$. Different scenarios are developed to explain such performance [6]-[8]. However, explanations on receiving properties of the Yagi-Uda is specific to the resonant frequency, and $\eta_{a}$ has not been explored outside resonance region. Also, $\check{\eta}_{a}$ has not been reported for this antenna so far. Here, we report the generalised absorption efficiency of a YagiUda antenna as 0.11 . We also report variation of absorption efficiency $\eta_{a}$ with frequency for this antenna.

\section{Generalised Absorption EFFiCIENCY}

The generalised absorption efficiency $\check{\eta}_{a}$, defined in [1], is a figure of the absorbed power in the load of the receiving antenna to the total extinct power by the antenna obstacle. This can be written as:

$$
\check{\eta}_{a}=\frac{\int_{0}^{\infty} \sigma_{a} / k^{2} d k}{\int_{0}^{\infty} \sigma_{e x t} / k^{2} d k}=\frac{\int_{0}^{\infty} \sigma_{a} d \lambda}{\int_{0}^{\infty} \sigma_{e x t} d \lambda},
$$

where $\sigma_{a}$ and $\sigma_{\text {ext }}$ are absorption and extinction cross sections of the antenna, respectively. The extinction cross section of an arbitrary object is the sum of the absorbed and scattered

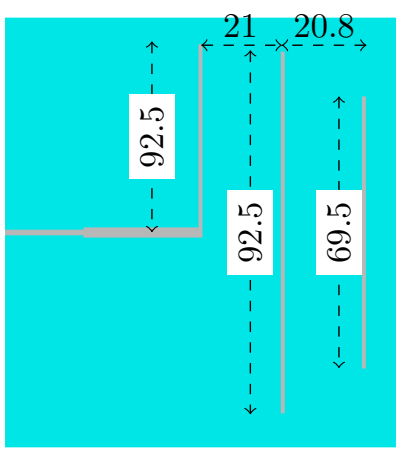

(a) Front

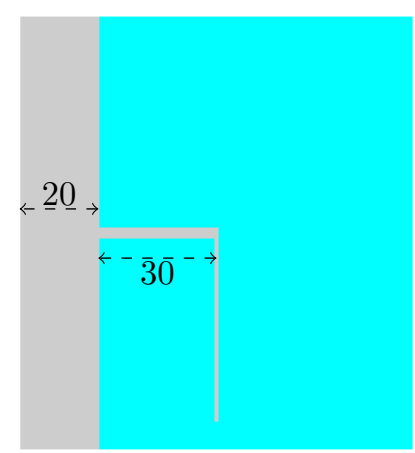

(b) Back
Fig. 1: Geometry of the Yagi-Uda antenna (all dimensions are in $\mathrm{mm}$ ).

cross sections as well as a loss term (if any lossy materials are present). It is illustrated in [1] that the maximum $D / Q$ is directly related to $\check{\eta}_{a}$

$$
\frac{D}{Q} \leq \frac{k^{3}}{2 \pi} \check{\eta}_{a}\left(\gamma_{e}+\gamma_{m}\right),
$$

where $\gamma_{\mathbf{e}}$ and $\gamma_{m}$ are the static electric and magnetic polarizability of the antenna. A numerical method to calculate polarizability of the arbitrary 3D objects is reported in [9].

As pointed in [3], there are two methods to calculate $\check{\eta}_{a}$. First method is to directly calculate $\sigma_{a}, \sigma_{s}$ from a broadband receiving antenna simulations. Another method is to write $\sigma_{a}$ as $\lambda^{2} G /(4 \pi)$ and perform integration. The all-spectrum extinction cross section is found from the forward scattering sum rule [10]

$$
\int_{0}^{\infty} \sigma_{e x t} d \lambda=\pi^{2}\left(\gamma_{e}+\gamma_{m}\right) .
$$

It should be noted that the generalized absorption efficiency $\check{\eta}_{a}$ should not be confused with the absorption efficiency $\eta_{a}$ which is defined as the ratio of the absorption to extinction cross section of a receiving antenna $\left(\sigma_{a} / \sigma_{\text {ext }}\right)$. We also emphasize that $\check{\eta}_{a}$ is not simply an integration over $\eta_{a}$.

\section{YAGI-UDA ANTENNA}

A planar Yagi-Uda antenna was designed using CST MW stuido for the studies in this paper. The structure includes a 


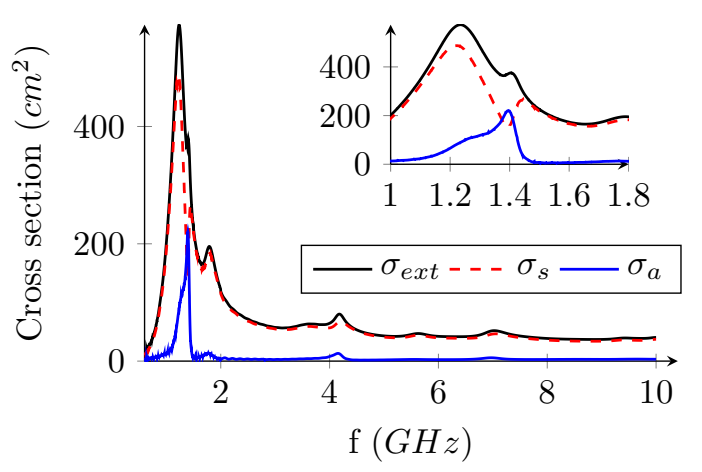

Fig. 2: Frequency sweep of absorption $\sigma_{a}$, scattering $\sigma_{s}$ and extinction cross secions $\sigma_{\text {ext }}=\sigma_{a}+\sigma_{s}$

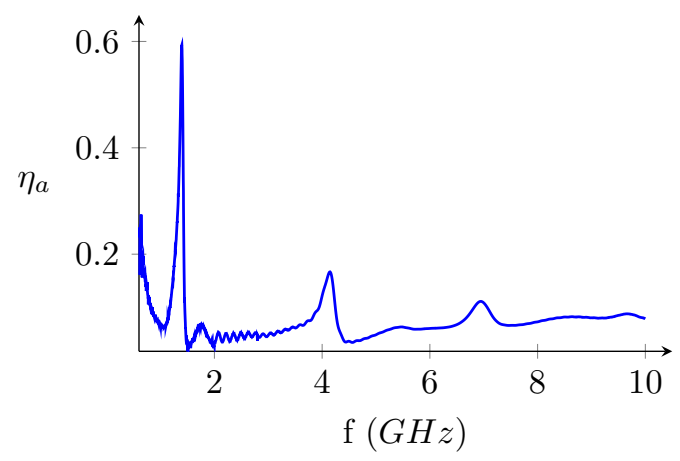

Fig. 3: Absorption efficiency $\eta_{a}$ versus frequency $\left(\eta_{a}\right.$ is different from $\check{\eta}_{a}$ ).

driving element and two directors printed on the dielectric substrate with $\epsilon_{0}=2.31$. A transition transmission line connects the arms of the driving element to the microstrip feed (see Fig. 1). The width of all radiating elements are $1 \mathrm{~mm}$. The antenna resonates at $1.4 \mathrm{GHz}$.

\section{REsults}

Figure 2 shows the variation of the antenna cross sections over the frequency range of $0.6-10 \mathrm{GHz}$. The behaviour of the receiving antenna near the resonance is illustrated in the small plot in Fig. 2. At the resonant frequency $(1.4 \mathrm{GHz})$, the absorption cross section $\sigma_{a}$ reaches the absolute maximum of the $221 \mathrm{~mm}^{2}$ while $\sigma_{s}$ has a local mimimum of the $151 \mathrm{~mm}^{2}$. Apart from the neighbourhood of resonant frequency, $\sigma_{a}$ is very low which means the antenna absorbs minimal energy from the incoming plane wave. The scattering cross section $\sigma_{s}$, on the other hand, stays close to the extinction cross section of the antenna (except from the resonance). Antenna cross sections have less variations at higher frequencies.

The variation of the absorption efficiency $\eta_{a}$ with frequency is shown in Fig. 3. The maximum absorption efficiency $\eta_{a}$ of the antenna is 0.59 which is achieved at $1.4 \mathrm{GHz}$. This high value of $\eta_{a}$ is only observed at the resonant frequency and it is less than 0.20 everywhere else.

The generalized absorption efficiency $\check{\eta}_{a}$ is calculated by separate integrations over the absorbed and scattered powers (cross sections). By doing that for the curves of Fig. 2, therefore, $\check{\eta}_{a}$ is found to be $\mathbf{0 . 1 1}$. This seems to be acceptable as the receiving spectrum of Yagi-Uda antenna is dominated by scattering from parasitic elements and the main radiator.

In addition to Yagi-Uda, electreically small meanderline $(\boldsymbol{k} \boldsymbol{a}<\mathbf{0 . 3})$ also reported with low $\check{\boldsymbol{\eta}}_{\boldsymbol{a}}$ [3]. However, various dipole, folded-dipole, spherical helix antennas are reported in [2, Table.1] with a generalized absorption efficiency of approximately $0.4-0.5$.

\section{CONClusion}

The generalized absorption efficiency $\check{\eta}_{a}$ of a Yagi-Uda antenna is reported in this paper. The calculations show that $\check{\eta}_{a}$ is 0.11 for this antenna. This is due to the fact that, at each frequency outside resonance band, Yagi-Uda absorbs less than $20 \%$ of extinct power from incoming wave. Therefore, ratio of the total absorbed power to the total extinct power $\left(\check{\eta}_{a}\right)$ comes down to 0.11 . We also illustrated the variation of the absorption efficiency over the frequency.

\section{ACKNOWLEDGEMENT}

The research leading to these results is partially funded by a grant from Australian Research Council DP130102098.

\section{REFERENCES}

[1] M. Gustafsson, C. Sohl, and G. Kristensson, "Physical limitations on antennas of arbitrary shape," Proc. R. Soc. A, vol. 463, no. 2086, pp. 2589-2607, 2007.

[2] M. Gustafsson, M. Cismasu, and S. Nordebo, "Absorption efficiency and physical bounds on antennas," Int. J. Antennas Propag., 2010.

[3] M. Shahpari, D. V. Thiel, and A. Lewis, "An investigation into the Gustafsson limit for small planar antennas using optimisation," IEEE Trans. Antennas Propag., DOI: 10.1109/TAP.2013.2290794.

[4] R. Collin, "Limitations of the thevenin and norton equivalent circuits for a receiving antenna," IEEE Antennas Propag. Mag., vol. 45, no. 2, pp. $119-124,2003$.

[5] I. Liberal and R. Ziolkowski, "Analytical and equivalent circuit models to elucidate power balance in scattering problems," IEEE Trans. Antennas Propag., vol. 61, no. 5, pp. 2714-2726, 2013.

[6] J. Andersen and R. Vaughan, "Transmitting, receiving, and scattering properties of antennas," Antennas and Propagation Magazine, IEEE, vol. 45, no. 4, pp. 93-98, 2003.

[7] S. Best and B. Kaanta, "A tutorial on the receiving and scattering properties of antennas," IEEE Antennas Propag. Mag., vol. 51, no. 5, pp. 26-37, 2009.

[8] H. Steyskal, "On the power absorbed and scattered by an antenna," IEEE Antennas Propag. Mag., vol. 52, no. 6, pp. 41-45, 2010.

[9] M. Shahpari, D. V. Thiel, and A. Lewis, "Polarizablity of 2D and 3D conducting objects using method of moments," ANZIAM Journal, vol. 54, pp. C446-C458, 2013.

[10] C. Sohl, M. Gustafsson, and G. Kristensson, "Physical limitations on broadband scattering by heterogeneous obstacles," J. Phys. A-Math. Theor, vol. 40, no. 36, p. 11165, 2007. 